\title{
Low-complexity hybrid QRD-MCMC MIMO detection
}

\author{
Ronghui Peng ${ }^{\dagger}$, Koon Hoo Teo ${ }^{\ddagger}$, Jinyun Zhang ${ }^{\ddagger}$ and Rong-Rong Chen ${ }^{\dagger}$ \\ ${ }^{\dagger}$ Electrical and Computer Engineering, University of Utah, Salt Lake City, UT 84112 \\ ${ }^{\ddagger}$ Mitsubishi Electric Research Laboratories, Cambridge, MA 02139 \\ $\dagger$ \{peng,rchen $\} @$ eng.utah.edu, ${ }^{\ddagger}\{$ Teo, Jzhang $\} @$ MERL.com
}

\begin{abstract}
In this paper, we propose a novel hybrid QRDMCMC MIMO detector that combines the features of a QRD-M detector and a Markov chain Monte Carlo (MCMC) detector. The QRD-M algorithm is applied first to obtain initial estimates of the transmitted signal vector. Subsequently, the QRD-M estimate is used to initialize one of the Gibbs samplers for MCMC detection. The MCMC detection reduces the $M$ parameter required by the QRD-M detector, while the QRD-M initialization effectively alleviates the well-known high-SNR problem in existing MCMC detectors. Performance of the QRD-M/MCMC detector is examined under both an idealized MIMO channel with perfect channel side information (CSI) and a practical IEEE 802.16e MIMOOFDMA system with imperfect CSI. Numerical results show that, compared to the stand-alone QRD-M or MCMC detectors, the QRD-MCMC detector achieves superior performance at a reduced complexity.
\end{abstract}

\section{INTRODUCTION}

The multiple-input multiple-output (MIMO) technology is one of most significant advancement in the past decade. In this work, we study the detection problem of spatial multiplexing MIMO system. The exponentially increasing complexity of the optimal maximal a posterior (MAP) detector motivates a significant amount of research in recent years to develop lowcomplexity MIMO detectors. Most of research work can be classified as four categories: 1) Linear detectors, such as, zero forcing (ZF) or minimum mean square error (MMSE) detectors; 2) Interference cancelation detectors, such as, VerticalBell Labs Layered Space Time (V-BLAST) [1]; 3) Tree search detectors, such as, sphere decoding [2] or QR decomposition with M algorithm (QRD-M) [3] detectors; 4) Markov chain Monte Carlo (MCMC) detector [4]. Linear detectors usually have lower complexity at the cost of performance degradation. Tree search detectors theoretically achieve optimal performance by selecting a large sphere (sphere decoding) or the $M$ parameter (QRD-M). However, the complexity of these detectors may become prohibitive at low SNR [5]. The MCMC detector, on the other hand, works well at low SNR, but encounters some problems at high SNR [6].

In this paper, we propose a new MIMO detector which is a combination of QRD-M and MCMC. We refer to it as the QRD-M initialized MCMC (QRD-MCMC) detector. With the aid of QRD-M, the high SNR problem of existing MCMC detectors is alleviated. In addition, compared to the

This work is supported in part by Mitsubishi Electric Research Laboratories and by NSF under grant ECS-0547433.

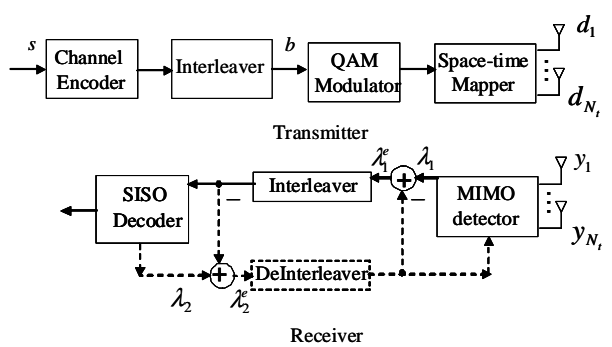

Fig. 1. MIMO spatial multiplexing with SDD and JDD (dash line) system model.

stand-alone QRD-M detector, QRD-MCMC requires a smaller $M$ parameter which leads to a reduced complexity. Our simulation results show that QRD-MCMC can achieve good performance for a wide range of SNR. Furthermore, through a detailed complexity analysis, we show that the QRD-MCMC detector achieves better performance than existing detectors with reduced complexity.

The remainder of the paper is organized as follows. In Section II, we introduce the system model. Section III includes a detailed description of the proposed QRD-MCMC detector. Complexity analysis is given in IV. Simulation results of the QRD-MCMC detector are presented in Section V for an idealized MIMO channel with perfect CSI, and in Section VI for a practical IEEE 802.16e downlink orthogonal frequencydivision multiple access (OFDMA) system with imperfect CSI. Conclusions are given in Section VII.

\section{SYSTEM DESCRIPTION}

We first describe the system model for a point-to-point MIMO channel. As shown in Fig. 1, a sequence of binary information bits $\left\{s_{i}\right\}$ is first encoded by a channel encoder of rate $R$. The interleaved coded bit sequence $\left\{b_{i}\right\}$ is then mapped to a sequence of QAM symbols. The modulated symbol stream is then partitioned to $N_{t}$ spatial streams that are transmitted through $N_{t}$ transmit antennas. At the receiver side, MIMO detection and channel decoding are performed to recover the transmitted bit sequence. In a system that performs separate detection and decoding (SDD), the MIMO detector generates $\lambda_{1}^{e}$, which is the extrinsic log-likelihood ratio (LLR) of each transmitted bit, based on $\mathbf{y}$ and pass them to the soft-input soft-output (SISO) channel decoder for channel decoding. For a joint detection and decoding (JDD) system, 


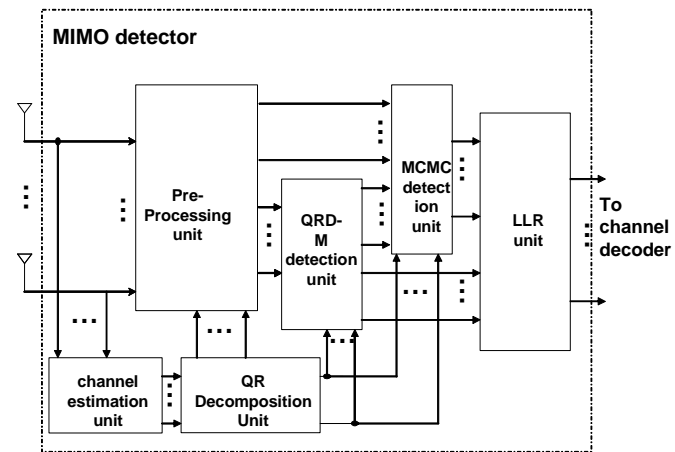

Fig. 2. A block diagram of QRM-MCMC detector.

the channel decoder performs one of multiple iterations of channel decoding to generate the improved LLRs, denoted by $\lambda_{2}^{e}$, to be passed back to the MIMO detector for joint iterative detection and decoding.

The point-to-point MIMO channel model is given by

$$
\mathbf{y}=\mathbf{H d}+\mathbf{n}
$$

where $\mathbf{d} \in C^{N_{t}}$ is the transmitted signal vector, $\mathbf{y} \in C^{N_{r}}$ is the received vector and $\mathbf{H} \in C^{N_{r} \times N_{t}}$ is the channel matrix. with independent and circular complex Gaussian distributed entries. $\mathbf{n}$ is an $N_{r} \times 1$ noise vector whose entries are zero mean independent, circular complex Gaussian random variable with variance $\sigma^{2}=N_{0} / 2$.

\section{QRD-MCMC MIMO DETECTION}

In this section, we present a novel MIMO detector that utilizes both QRD-M and MCMC detection algorithms.

Given $\mathbf{y}$, the extrinsic LLR of a particular bit $b_{k}$ is given by

$$
\lambda_{1, k}^{e}=\ln \frac{P\left(b_{k}=+1 \mid \mathbf{y}\right)}{P\left(b_{k}=-1 \mid \mathbf{y}\right)}=\ln \frac{\sum_{\mathbf{b}_{-k}} P\left(b_{k}=+1, \mathbf{b}_{-k} \mid \mathbf{y}\right)}{\sum_{\mathbf{b}_{-k}} P\left(b_{k}=-1, \mathbf{b}_{-k} \mid \mathbf{y}\right)}
$$

where $\mathbf{b}_{-k}=\left(b_{1}, \cdots b_{k-1}, b_{k+1}, \cdots, b_{N_{t} M_{c}}\right) ; b_{i} \in\{-1,1\}$, and $M_{c}$ is the number of bits in each constellation symbol. The optimal MAP detector operates according to (2) which requires an exponential complexity.

To reduce complexity, suboptimal detectors such as the tree search detector (e.g. QRD-M detector and sphere decoder) and the MCMC detector adopt different algorithms to identify a small set of most likely transmitted vectors based on which the LLRs are computed. Both the QRD-M detector and the MCMC detector, however, have certain limitations. It is known that, while the tree search detector is effective at high SNR, its complexity becomes prohibitive at low SNR [5]. The MCMC detector, on the other hand, is effective at low SNR, but experiences some problems at high SNR [6]. In this work, we propose a hybrid QRD-MCMC detector that reaps the benefits of both detectors. A diagram of the QRD-MCMC detector is shown in Fig. 2. The main idea is to perform QRD$\mathrm{M}$ detection first, using a smaller $M$ parameter, followed by MCMC detection. The most likely transmitted vector found by the QRD-M algorithm is used as one of the initial vectors to initiate MCMC detection. The QRD-M and MCMC algorithms complement each other in that the QRD-M ensures that the MCMC starts from the proper initial vector at high SNR, therefore alleviating the high SNR problem; and the MCMC detector effectively reduces the $M$ parameter required by the QRD-M algorithm, hence reducing the high complexity of QRD-M detector at low SNR. We note that in related work [6], a MMSE initialized MCMC (MMSE-MCMC) is proposed to mitigate the high SNR problem of the previously proposed random initialized MCMC (RND-MCMC) [4]. Our results in Section $\mathrm{V}$ demonstrate that the proposed QRD-MCMC outperforms both MMSE-MCMC and RND-MCMC over a wide range of SNR at reduced complexity.

Detailed implementation of the QRD-MCMC detector is described as follows:

First, we perform the QR decomposition over the channel matrix $\mathbf{H}$ such that $\mathbf{H}=\mathbf{Q R}, \mathbf{Q} \in C^{N_{r} \times N_{r}}$ is a unitary matrix and

$$
\mathbf{R}=\left[\begin{array}{c}
\mathbf{T} \\
\mathbf{0}_{N_{r}-N_{t}, N_{t}}
\end{array}\right]
$$

where $\mathbf{T} \in C^{N_{t} \times N_{t}}$ is an upper-triangular matrix. Then we have

$$
\mathbf{r}=\mathbf{Q}^{H} \mathbf{y}=\mathbf{Q}^{H} \mathbf{H} \mathbf{d}+\mathbf{Q}^{H} \mathbf{n}=\mathbf{R} \mathbf{d}+\mathbf{Q}^{H} \mathbf{n}
$$

where $(\cdot)^{H}$ denotes the Hermitian operator,

For ease of disposition, we assume that $N_{t}=N_{r}$ (thus $\mathbf{R}=\mathbf{T}$ ) hereafter. While most previous work on QRD-M considers SDD systems, here we present a SISO version of the QRD-M that is directly applicable to the JDD systems. We illustrate the QRD-M algorithm using a tree shown in Fig. 3, assuming that $N_{t}=N_{r}=4, M_{c}=2$ and $M=2$. The node at the top represents the transmitted symbol $d_{4}$. Since $d_{4}$ may take 4 different values, there are 4 branches connected to the $d_{4}$ node, each representing a possible value of $d_{4}$. These branches are connected to the nodes $d_{3}$ at the next layer, etc. Hence, each path in the tree corresponds to a transmitted sequence $\left(d_{4}, d_{3}, d_{2}, d_{1}\right)$. We define the path metric associated with each path as

$$
\begin{aligned}
|\omega|^{2} & =\sum_{k=1}^{N_{t}}\left|r_{k}-\sum_{l=k}^{N_{t}} t_{k, l} d_{l}\right|^{2}-2 \sigma^{2} \sum_{k=1}^{N_{t}} p\left(d_{k}\right) \\
& =\sum_{k=1}^{N_{t}}\left|\left(r_{k}-t_{k, k} d_{k}\right)-\sum_{l=k+1}^{N_{t}} t_{k, l} d_{l}\right|^{2}-2 \sigma^{2} \sum_{k=1}^{N_{t}} p\left(d_{k}\right)
\end{aligned}
$$

where $r_{k}, t_{k, l}, d_{l}$ are the entries of $\mathbf{r}, \mathbf{T}, \mathbf{d}$, respectively, and $p\left(d_{k}\right)$ is prior probability of symbol $d_{k}$ provided by SISO decoder.

The QRD-M reduces the tree search complexity by keeping only $M$ branches that have the smallest accumulated path metric $\lambda_{i}$ at each tree level $i$. For example, as shown in Fig. 3, at level 1 , only 2 out of 4 possible $d_{4}$ with smallest $\lambda_{4}=\left|r_{4}-t_{4,4} d_{4}\right|^{2}-2 \sigma^{2} p\left(d_{4}\right)$ associated with $d_{4}$ are selected. With each selected $\hat{d}_{4}$, we update $r_{k}=r_{k}-t_{k, 4} \hat{d}_{4}, 1 \leq k \leq 3$ which are used to compute the accumulated path metric $\lambda_{3}$ at the next level. This computation is carried out level by level until level 4 to yield $\lambda_{1}$ which equals the path metric defined in (4). Finally, we obtain two paths with the smallest path 


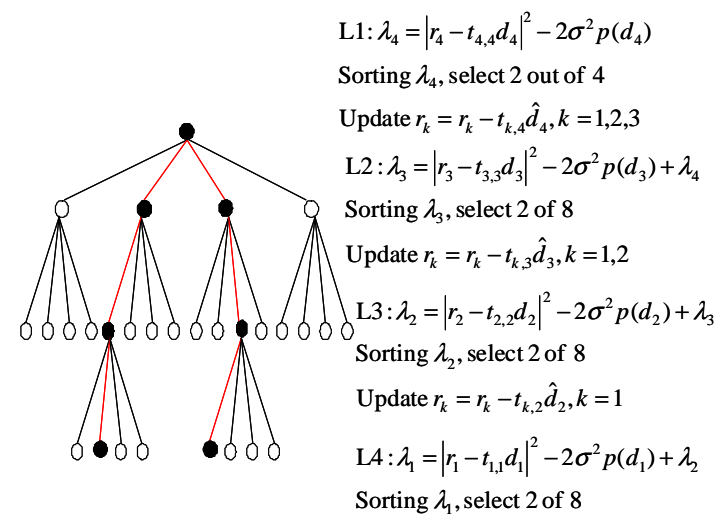

Fig. 3. A QRD-M detector with $N_{t}=4, M_{c}=4, M=2$. $\lambda_{i}$ denotes the accumulated path metric corresponding to the transmitted sequence $\left(d_{4}, d_{3}, \cdots, d_{1}\right)$.

metric. The one signal vector with the minimum metric is use to initialize the MCMC detection.

Next, for completeness we include a brief description of the RND-MCMC detector of [4]. The MCMC detector uses Gibbs sampler to generate a list of $L$ most likely transmitted vectors, where $L$ is the number of iteration in the Gibbs sampler. The Gibbs sampler works follows:

$$
\begin{aligned}
& \text { Generate initial } \mathbf{b}^{(0)} \\
& \text { for } n=1 \text { to } L \\
& \text { generate } b_{0}^{(n)} \text { from distribution } \\
& \quad p\left(b_{0}=b \mid b_{1}^{(n-1)}, b_{2}^{(n-1)}, \cdots, b_{N_{t} M_{c}-1}^{(n-1)} \mathbf{y}\right) \\
& \text { generate } d_{1}^{(n)} \text { from distribution } \\
& \quad p\left(b_{1}=b \mid b_{0}^{(n)}, b_{2}^{(n-1)}, \cdots, b_{N_{t} M_{c}-1}^{(n-1)} \mathbf{y}\right) \\
& \quad \vdots \\
& \text { generate } b_{N_{t} M_{c}-1}^{(n)} \text { from distribution } \\
& \quad p\left(b_{N_{t} M_{c}-1}=b \mid b_{0}^{(n)}, b_{1}^{(n)}, \cdots, b_{N_{t} M_{c}-2}^{(n)}, \mathbf{y}\right) \\
& \text { end for }
\end{aligned}
$$

where

$$
\begin{aligned}
& p\left(b_{i}=b \mid b_{0}^{(n)}, \cdots b_{i-1}^{(n)}, b_{i+1}^{(n-1)}, \cdots, b_{N_{t} M_{c}-1}^{(n-1)} \mathbf{y}\right) \propto \\
& \quad p\left(\mathbf{y} \mid b_{0}^{(n)}, \cdots b_{i-1}^{(n)}, b_{i}=b, b_{i+1}^{(n-1)}, \cdots, b_{N_{t} M_{c}-1}^{(n-1)},\right) p\left(b_{i}=b\right)
\end{aligned}
$$

Here $\mathbf{b}^{(0)}$ is the initial bit sequence, $b_{i}^{(n)}$ is the $i$-th bit generated during the $n$-th iteration.

In [4], it is shown that better performance can be achieved by running multiple Gibbs samplers in parallel. In the QRDMCMC detector, we assume that, out of $D$ parallel Gibbs samplers, one is initialized using the bit vector with the minimum path metric found by the QRD-M algorithm, and the remaining $D-1$ Gibbs samplers use random initialization. We will show that the QRD-M initialization effectively helps the Gibbs sampler to converge to most likely transmitted vectors, thus leads to better performance.

Once the MCMC detector produces the set of most likely transmitted vectors, denoted by $\mathcal{A}$, the output LLRs are computed by applying the max-log algorithm over $\mathcal{A}$.

$$
\lambda_{1, k}^{e} \approx \ln \frac{\max _{-k \in \mathcal{A}_{-k}} P\left(b_{k}=+1, \mathbf{b}_{-k} \mid \mathbf{y}\right)}{\max _{-k \in \mathcal{A}_{-k}} P\left(b_{k}=-1, \mathbf{b}_{-k} \mid \mathbf{y}\right)}
$$

where $\mathcal{A}_{-k}$ denotes the set of vectors in $\mathcal{A}$ with its $k$-th element deleted.

\section{COMPLEXITY ANALYSIS}

Although QRD-MCMC detector employs both QRD-M and MCMC algorithms, it can achieve better performance than the stand-alone QRD-M or MCMC detectors at reduced complexity due to the following reason:

- With the aid of QRD-M initialization, the MCMC detector starts from good initial vectors, which reduces the number of required parallel Gibbs samplers $D$ and the number of iterations $L$ per Gibbs sampler.

- Due to the use of MCMC, a small $M$ is sufficient for the QRD-M detection, leading to reduced complexity and delay.

- Due to the QR decomposition of the channel matrix, the computation of path metric in MCMC detection is reduced at least by half by using (4) instead of

$$
|\omega|^{2}=\sum_{k=1}^{N_{r}}\left|y_{k}-\sum_{l=1}^{N_{t}} h_{k, l} d_{l}\right|^{2}-2 \sigma^{2} \sum_{k=1}^{N_{t}} p\left(d_{k}\right)
$$

Furthermore, implementation of the Gibbs sampler involves computation of the path metric of a vector $\mathbf{d}^{\prime}$ from the path metric of $\mathbf{d}$, where $\mathbf{d}^{\prime}$ and $\mathbf{d}$ differs by one bit in symbol $d_{m}$. Next, we propose an efficient method to compute the path metric that requires only $\left(N_{t}+1\right)$ multiplications.

Let us express the path metric associated with $\mathbf{d}$ as $|\omega|^{2}=$ $\sum_{k=0}^{N_{t}} \gamma_{k}$, where $\gamma_{k}=\left|\omega_{k}\right|^{2}$ and $\omega_{k}=r_{k}-\sum_{l=k}^{N_{t}} t_{k, l} d_{l}$. Then the path metric for $\mathbf{d}^{\prime}$, expressed in terms of $\omega^{\prime}, \omega_{k}^{\prime}$ and $\gamma_{k}^{\prime}$ can be computed as follows:

$$
\begin{aligned}
& \text { for } k=1: m \\
& \qquad \begin{aligned}
\omega_{k}^{\prime} & =\omega_{k}+t_{k, m} d_{m}-t_{k, m} d_{m}^{\prime}=\omega_{k}-\phi ; \\
\gamma_{k}^{\prime} & =\gamma_{k}-2 \operatorname{Re}\left(\omega_{k} \phi^{*}\right)+|\phi|^{2}
\end{aligned} \\
& \text { end } \\
& \qquad\left.\omega^{\prime}\right|^{2}=\sum_{k=0}^{N_{t}} \gamma_{k}^{\prime}-2 \sigma^{2}\left[p\left(d_{m}^{\prime}\right)-p\left(d_{m}\right)\right]
\end{aligned}
$$

where $\operatorname{Re}(\cdot)$ is the real part of a complex number, and $\phi=$ $\left\{t_{k, m} d_{m}-t_{k, m} d_{m}^{\prime}\right\}$. Because $\gamma_{k}$ and $|\phi|^{2}$ are predetermined, only $\left(N_{t}+1\right)$ multiplications are required to compute the new path metric, which reduces the complexity of the QRD-MCMC detector. In particular, when $N_{t}=N_{r}$, the complexity of QRD-MCMC is about half of the complexity of RND-MCMC due to the $\mathrm{QR}$ decomposition. The complexity order of the QRD-MCMC is $O\left(N_{t}^{2} D L M_{c}\right)$.

In Table I, we compare the complexity of QRD-M, RNDMCMC, and QRD-MCMC in terms of the number of addition, comparison, and multiplication required for generating the set of likely transmitted vectors $\mathcal{A}$ and for computing the path metric. The complexity of $\mathrm{QR}$ decomposition, of the order of $O\left(4 N_{t}^{3}\right)$, is not considered here because it is 
TABLE I

COMPLEXITY OF THREE DETECTORS

\begin{tabular}{|c||c|c|c|}
\hline & QRD-M & RND-MCMC & QRD-MCMC \\
\hline \hline Add & $L_{c}\left\{1+2 N_{t}+M\left(N_{t}^{2}-1\right)\right\}$ & $\left(1+N_{t}\right) N_{t} D\left(3 L M_{c}+1\right)$ & $N_{a q}+N_{a m} / 2$ \\
\hline Compare & $L_{c}\left\{9 M N_{t}-5 N_{t}+N_{t}(M-3) \log _{2} M / 2\right\}$ & & $N_{c q}$ \\
\hline Mult & $2 L_{c}\left\{1+\left(N_{t}-1\right) M\right\}$ & $2\left(1+N_{t}\right) N_{t} D L M_{c}$ & $N_{m q}+N_{m m} / 2$ \\
\hline
\end{tabular}

In Table I, $D$ denotes the number of parallel Gibbs samplers, $L$ denotes the number of iterations per Gibbs sampler, $L_{c}$ denotes the number of modulated symbols, $M_{c}=\log _{2} L_{c}$ denotes number of bits per modulated symbol, $N_{a q}, N_{a m} N_{m q}, N_{m m}$ denote the number of additions and multiplications for QRD-M and MCMC, $N_{c q}$ denotes the number of comparisons for QRD-M.

negligible compared to the computation of path metrics. The corresponding complexity numbers for the detectors used in our simulations are given in Table II. The QRD-MCMC has a lower complexity than QRD-M, while RND-MCMC has the highest complexity.

TABLE II

COMPLEXITY COMPARISON OF THREE DETECTORS

\begin{tabular}{|c||c|c|}
\hline QRD-M & $4 \times 4 \mathrm{M}=32$ & 50256 \\
\hline & $8 \times 8 \mathrm{M}=32$ & 127344 \\
\hline RND-MCMC & $4 \times 4=8 L=5$ & 73760 \\
\hline & $8 \times 8 D=10 L=5$ & 331920 \\
\hline QRD-MCMC & $4 \times 4 M=9 D=6 L=5$ & 41498 \\
\hline & $8 \times 8 M=8 D=5 L=5$ & 114284 \\
\hline
\end{tabular}

In Table II,The complexity for 4x4 (antenna) and 8x8 16QAM system are compared for three detectors. The total operations are computed by considering one multiplication as 10 additions and one comparison as 0.5 additions.

\section{Performance of Mimo Channels With PERfect CSI}

In this section, the simulation result and comparison with other state-of-art detectors are given. In all simulations, we assume Rayleigh fading channel and the receiver knows the perfect CSI.

First, for various detectors, we examine the quality of the set of likely transmitted vectors $\mathcal{A}$ by plotting the minimum path distance over $\mathcal{A}: \min _{d^{\prime} \in \mathcal{A}}\left|\mathbf{y}-\mathbf{h} \mathbf{d}^{\prime}\right|^{2}$ and comparing it with the true distance $\left(|\mathbf{y}-\mathbf{h d}|^{2}\right)$, where $\mathbf{d}$ is the actual transmitted vector. As shown in Fig. 4, QRD-MCMC has the smallest average distance than the other two detectors for a wide range of SNR. This implies that QRD-MCMC produces the output LLRs with the best quality. When compared with the true distance, we note that at low SNR, the minimum distance over $\mathcal{A}$, produced by various detectors, are smaller than that of the true distance. This is because when the signal is corrupted by larger noise, the true distance is likely to be larger than the minimum distance found by the detectors. As SNR increases, the true distance becomes more likely the minimum distance, hence it would lower bound the minimum distance found by the detectors.

Next, we examine the performance of coded systems employing various MIMO detectors. In all simulations, a rate 1/2 IEEE 802.16e LDPC code [9] with code length 2304 is used. In Fig. 5, we compare the performance of QRD-M, RND-MCMC and QRD-MCMC detectors in 4 transmitters and 4 receivers 16QAM system for SDD (solid line) and JDD (dash line) system. For SDD system, one MIMO detection

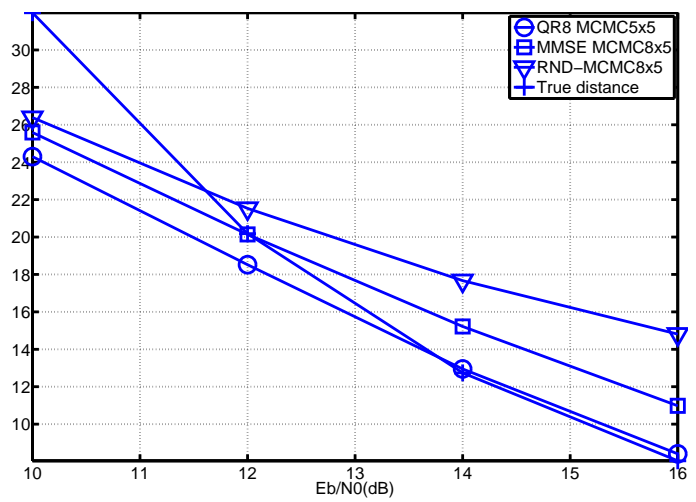

Fig. 4. Average minimum path metric for different detectors in $8 \times 8$ 16QAM SDD system

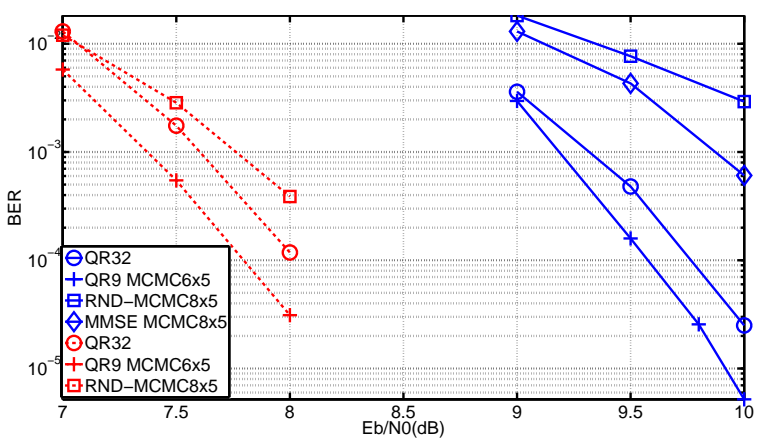

Fig. 5. Performance comparison of 4x4 16QAM SDD and JDD systems.

is performed followed by the 40 LDPC iterations. For JDD system, 8 outer iterations of joint detection and decoding are performed and each outer iteration consist of one MIMO detection followed by 5 LDPC iterations. The number of parallel Markov chains $D$ and the number of iteration $L$ per Markov chain in RND-MCMC and QRD-MCMC detector are denoted in the figures as MCMC $D \times L$. The $M$ parameter is denoted in figures as QR $M$. Here the parameters are selected so that QRD-MCMC has lower complexity than QRD-M (Table II). From Fig. 5, we can see the QRD-MCMC has the best performance and the RND-MCMC has the worst performance for both SDD and JDD systems. The gain of QRD-MCMC is more pronounced in a JDD system. Note within our simulation range, no error floor has been observed at high SNR (not shown in the figure) which confirms the effectiveness of QRD-MCMC over high SNR region.

In Fig. 6, we consider a 16QAM system with 8 transmit and receive antennas. It is shown that QRD-MCMC outperforms QRD-M by about $0.4 \mathrm{~dB}$ in a SDD system, and by about 


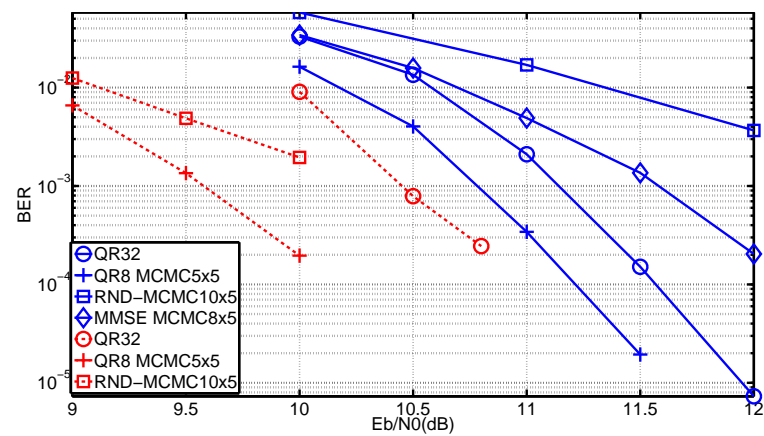

Fig. 6. Performance comparison of 8x8 16QAM SDD and JDD system

$1 \mathrm{~dB}$ in a JDD system. We also note that RND-MCMC performs worse than QRD-M in a SDD system but better in a JDD system. The performance of MMSE-MCMC for a SDD system is also plotted Fig. 6 which shows that, the utilization of MMSE to generate initial candidates achieves better performance than RND-MCMC, but still worse than QRD-MCMC.

\section{Performance of MiMO-OFDMA system with IMPERFECT CSI}

In this section, we apply the QRD-MCMC detector to a IEEE 802.16e downlink MIMO-OFDMA SDD system and examine the detector performance under imperfect CSI. A 2-D MMSE channel estimator is employed for channel estimation [10]. In turbo decoder, BCJR decoding with 15 iterations is performed. Detailed system parameters are given in Table III.

TABLE III

SYSTEM PARAMETERS

\begin{tabular}{|c|c|}
\hline Parameter & Value \\
\hline \hline Channel bandwidth & $10 \mathrm{MHz}$ \\
\hline Number of subcarriers & 1024 \\
\hline Subcarrier permutation & PUSC \\
\hline Cyclic prefix & $1 / 8$ \\
\hline Channel coding & Convolutional turbo codes \\
\hline Carrier frequency & $2500 \mathrm{MHz}$ \\
\hline Sampling frequency & $11.2 \mathrm{MHz}$ \\
\hline Multipath channel & ITU VehA \\
\hline MS speed & $120 \mathrm{~km} / \mathrm{hr}$ \\
\hline
\end{tabular}

We compare the performance of the QRD-MCMC detector with the optimal max-log MAP detector. Fig. 7 shows that, under imperfect CSI, the QRD-MCMC $6 \times 5$ detector performs only about $0.5 \mathrm{~dB}$ worse than the max-log MAP detector. In order to see the effect of channel estimation error on system performance, we also plot the performance curves (shown as the dash lines) of these detectors assuming that perfect CSI is available at the receiver. In this case, QRD-MCMC performs very close to the max-log MAP detector. However, we observe that the performance gap between the case of perfect CSI and imperfect CSI is more than $7 \mathrm{~dB}$, possibly due to the fact that under the high mobility scenario considered here, the pilots in IEEE 802.16e system are insufficient to track the variation of the channel, and the channel estimation error rather than white noise dominates performance. We expect

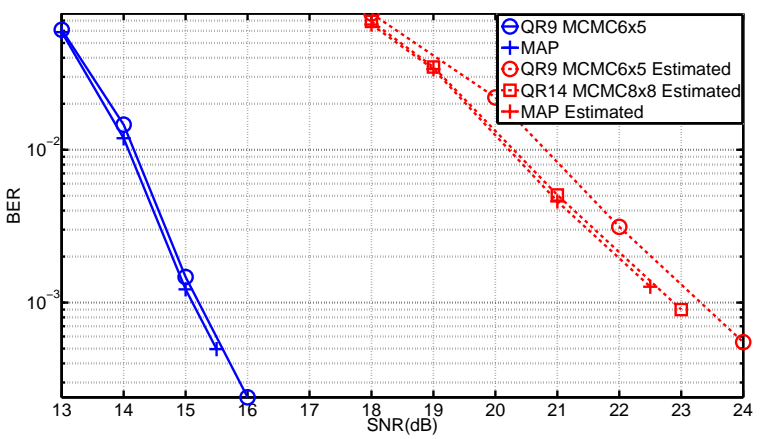

Fig. 7. Performance comparison of $4 \times 4$ 16QAM MIMO-OFDMA system using $R=1 / 2$ IEEE $802.16 \mathrm{e}$ convolutional turbo codes with perfect and estimated channel estimation.

that data-aided channel estimation may improve the quality of channel estimation thus leading to better performance.

\section{CONCLUSION}

In this paper, we develop a hybrid QRD-MCMC MIMO detector that exploits the advantages of both QRD-M and MCMC detection. Through a detailed complexity and performance analysis, we demonstrate that the QRD-MCMC detector yields superior performance than QRD-M and MCMC detector with substantial complexity saving. Furthermore, the high SNR problem of existing MCMC detectors is effectively alleviated in the QRD-MCMC due to proper initialization using the QRD-M algorithm. Application to a practical MIMO-OFDMA system shows QRD-MCMC performs closely to the optimal MAP detector under imperfect channel CSI. We conclude that QRD-MCMC is a good competing MIMO detector in terms of both the performance and complexity.

\section{REFERENCES}

[1] G. D. Golden, G. J. Foschini, R. A. Valenzuela, and P. W. Wolniansky, "Detection algorithm and initial laboratory results using V-BLAST space-time communication architecture," Electronic Letters, vol. 35, no. 5, pp. 14-16, Jan. 1999.

[2] B. M. Hochwald and S. ten Brink, "Achieving near-capacity on a multiple antenna channel," IEEE Trans. Commun., vol. 51, no. 3, pp. 389-399, Mar. 2003.

[3] K. J. Kim and J. Yue, "Joint channel estimation and data detection algorithms for MIMO-OFDM systems," in Thirty-Sixth Asilomar Conference on Signals, Systems and Computers, 2002, pp. 1857-1861.

[4] B. Farhang-Boroujeny, H. Zhu, and Z. Shi, "Markov chain Monte Carlo algorithms for CDMA and MIMO communication systems," IEEE Trans. Signal. Process., vol. 54, no. 5, pp. 1896-1909, May 2006.

[5] J. Jaldén and B. Ottersten, "On the complexity of sphere decoding in digital communications," IEEE Trans. Signal Process., vol. 53, no. 4, pp. 1474-1484, April 2005.

[6] X. Mao, P. Amini, and B. Farhang-Boroujeny, "Markov Chain Monte Carlo MIMO Detection Methods for High Signal-to-Noise Ratio Regimes," in Proc. Globecom, Washington, DC, USA, Nov. 2007, pp. 3979-3983.

[7] S. Chen, T. Zhang, and Y. Xin, "Relaxed K-Best MIMO Signal Detector Design and VLSI Implementation," IEEE Trans. VLSI systems, vol. 15, pp. 328-337, 2007.

[8] S. A. Laraway and B. Farhang-Boroujeny, "Implementation of a Markov Chain Monte Carlo Based Multiuser/MIMO Detector," in IEEE International Conference on Communications, ICC 2006, vol. 7, Istanbul, Turkey, June 2006, pp. 3088-3093.

[9] IEEE Std 802.16e-2005, approved Dec 2005, pub. Feb 2006.

[10] W. G. Jeon, K. H. Paik, and Y. S. Cho, "Two-dimensional pilot-symbolaided channel estimation for OFDM systems with transmitter diversity," IEICE Trans. Commun., vol. E85-B, pp. 840-844, Apr. 2002. 